\title{
The Virtual Liver Network: systems understanding from bench to bedside
}

\author{
"...systems biology is the logical step to follow on from the genome \\ program ... which now needs to be integrated into an holistic view. The natural \\ home for integration is ... a systems-wide view built on the strengths of \\ mathematical modeling and simulation strategies."
}

\begin{abstract}
Adriano Henney speaks to Hannah Coaker, Commissioning Editor. After achieving a $\mathrm{PhD}$ in medicine and spending many years in academic research in the field of cardiovascular disease, Adriano Henney was recruited by Zeneca Pharmaceuticals from a British Heart Foundation Senior Fellowship, where he led the exploration of new therapeutic approaches in atherosclerosis, specifically focusing on his research interests in vascular biology. Following the merger with Astra to form AstraZeneca, Henney became responsible for exploring strategic improvements to the company's approaches to pharmaceutical target identification and the reduction of attrition in early development, directing projects across research sites and across functional project teams in the USA, Sweden and the UK. This resulted in the creation of a new multidisciplinary department that focused on pathway mapping, modeling and simulation and supporting projects across research and development, which evolved into the establishment of the practice of systems biology within the company. Here, projects prototyped the application of mechanistic disease-modeling approaches in order to support the discovery of innovative new medicines, such as Iressa ${ }^{\circledR}$. Since leaving AstraZeneca, Henney has continued his interest in systems biology, synthetic biology and systems medicine through his company, Obsidian Biomedical Consulting Ltd. He now directs a major $€ 50$ million German national flagship program - the Virtual Liver Network - which is currently the largest systems biology program in Europe.
\end{abstract}

Q How did you become involved in the field of systems biology?

During my time at AstraZeneca, I was responsible for a global program that looked at novel approaches for reducing attrition in Phase II to Phase III studies, prototyping novel science in collaboration with drug discovery and occasionally development projects that trialed these new ideas. The program had six different projects within it, two of which were related to pathway analysis and mathematical modeling of cellular pathways. In short, during the prototyping phase, two key projects survived, and those were the ones involving pathway mapping and modeling, demonstrating that both of these areas had promise. In light of these results, we created a new department called 'Pathways', which combined both of these projects. Pathways had a strong multidisciplinary focus and consisted of mathematicians, engineers, physicists and experimentalists working together in a variety of ways in order to get a better view of how pathways operated in cells and how we could better understand their function. The strategy we employed was, to all intents and purposes, a systems biology approach, although we did not identify it as such at the time.

Pathways was one of the first industry departments to look at mechanistic modeling in discovery and development. Within the department, we worked very closely with both drug discovery and development teams and clinical teams in order to try out some ideas so as to demonstrate how modeling and simulation could help to give a different perspective from the way things were normally done in the discovery pipeline. Even though I was not a systems biologist by any means,

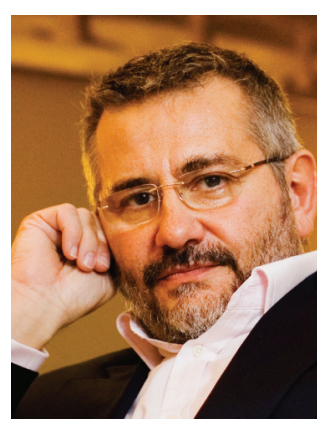

Adriano Henney Obsidian Biomedical Consulting Ltd, 34 Ploughmans Way, Macclesfield, Cheshire SK10 2UN, UK adriano.henney@obsidian-biomed.com 
it became very clear to me that we needed the tools that prevailed in engineering, particularly mathematical modeling, to help us reduce the complexity that we see in biological systems and to help us understand the dynamics of those biological systems. Typically, bioscientists do this by using omics approaches in order to generate a series of snapshots that serve as an indication of how things happen in biological systems over a period of time. The strength of mathematical modeling, however, is the capacity to look at the dynamics of interactions in a biological system by creating simulations, rather than comparing static snapshots of system outputs. It became very clear to me that this was the way forward if we were going to be able to begin to understand how biological systems work.

\section{Q Did your move into the systems biology} field feel like a natural progression from your academic studies?

My undergraduate degree was in microbiology, and following that, I did a PhD in medicine, which focused specifically on cardiovascular pathology. I have always had an interest in the mechanisms that underpin disease and my career has focused on broadening my understanding of them. Over time, I increasingly moved into the area of molecular genetics, and that gave me a broad introduction to the variety of techniques and approaches that ultimately helped me understand and further appreciate biological complexity.

Rather than being someone who is wedded to a particular discipline, technology or approach and whose aim is to become an expert in a specific subject, I like to gain a broader picture of the connection between basic science and medicine. Trying to understand how we can improve the communication between the bench and the bedside is something that has been central to my particular interests for a very long time.

Q Is there a specific area of the field that you are particularly excited about currently?

I think that the concept of being able to demonstrate the value of mathematical modeling and simulation in understanding complex biological systems is very exciting, and I speak as a nonmathematician! Increasingly, we are seeing examples of models being used to inform submissions to the US FDA. Merrimac Pharmaceuticals was recently, I believe, the first to use a systems biology approach to demonstrate Phase III success of a cancer drug. I am sure that instances such as this are going to increase and I am excited by the prospect of systems biology finally coming of age in industry. From my perspective, I am most excited about seeing real examples of systems biology's impact in the clinic; for example, the use of mathematical models in order to help with decision-making, which is something we are not far from attaining. There are many advances being made in the field and I believe that, in time, these approaches will become less science fiction and more science fact.

\section{Q It is widely believed that systems biology} has the potential to reduce attrition in early drug development $\&$ accelerate the discovery of innovative new drugs. Do you think that these are unrealistic expectations?

I have always been an advocate of not overhyping a new technology, as we know how this can build people's expectations and may cause huge damage if the promise is not realized. A problem that we are experiencing currently is that systems biology seems to have acquired a bit of a bad press and some people are simply no longer interested in what it has to offer, which I think is hugely unfortunate and is something we really have to try to rectify. The other problem is that the definition of systems biology is very broad and there are many who will interpret it in different ways, from statistical approaches, to interpreting snapshot data in omics, to the use of modeling and simulation for generating testable hypotheses. I favor the latter definition, which gives a very dynamic perspective by simulating a complex network and, in that sense, contrasts significantly with the snapshot approach.

\section{"...the problem lies in actually establishing [these systems] routinely in drug discovery and development."}

The other problem is the incorrect assumption that models of a biological system have to include absolutely everything, which is simply not the case. Models need to be sufficient to generate a faithful representation of the thing that they are modeling; if they do not acheive that at the first attempt, you enter into an iterative process between experimentation and modeling, until you eventually generate a model that is validated in that particular context. Mathematical models can be used in a variety of ways in order to tackle complex questions and aid decision-making broadly across the whole value chain of drug discovery and development. For example, they may be used to model particularly complex experiments in order to determine the priority areas you should be concentrating on, as opposed to carrying out a range of large, time-consuming experiments. They may also be applied in order to elucidate the interaction of a target and compound in a particular biological compartment by creating a 'fit for purpose' model that only addresses a specific question for those particular circumstances and no other. 
Essentially, it is a case of using the modeling toolbox to tackle complexity and to understand the dynamics of interactions, and I think these concepts are not widely understood. People assume that systems biology involves the generation of huge models of very complex systems, which take years to produce. When I was at AstraZeneca, however, we saw a number of examples of small, focused models that we created in a matter of a couple of months that were very detailed and very specific and helped us to address the particular question we were trying to answer. If you consider systems biology in this context, you can see how modeling and simulation can have a dramatic impact on the pipeline. A very interesting series of reports from PricewaterhouseCoopers that were published in 2007 and 2008 advocated for increased 'virtualization' - increased modeling and simulation - in order to do precisely that. The application of these systems is not rocket science and it is not novel - the problem lies in actually establishing it routinely in drug discovery and development.

\section{Q Systems biology draws on a variety of} disciplines \& requires close collaboration in order to ensure a dynamic approach to drug R\&D: what are the challenges of this drug discovery model? Of all places, the application of systems biology tools should not present that much of a challenge in the pharmaceutical industry because, after all, drug discovery and development involves multidisciplinary interactions and engagement from the start. In the pharmaceutical industry, you have people coming from all sorts of disciplines - let us take chemistry and biology as a starter. The concept of more than one discipline and expertise in a drug discovery project team is not alien, so it really should be less of a problem in industry compared with academia, for example, where things are a lot more compartmentalized and there are still issues with theoreticians and experimentalists finding the right language to understand one another. In academia, we see pigeonholing of disciplines and faculties, which makes cross-discipline and multidisciplinary training and development in an academic sense really quite a challenge. This is something that we touched on in a joint Academy of Medical Sciences and the Royal Academy of Engineering report on systems biology, for which I was a panel member of the committee chaired by Sir Collin Dollery from the Academy of Medical Sciences and Professor Richard Kitney from Imperial College London. In the report, we touched on how you ensure that individuals develop a multidisciplinary mindset and skill base, particularly for biologists, and improving their understanding of mathematical approaches.

The challenge in industry is less to do with adopting a multidisciplinary approach, because I think that this is broadly there already; rather, it is much more to do with the introduction of a novel or disruptive technology - disruptive in the sense that it changes the status quo or is unproven and certainly not reduced to practice sufficiently for routine application with confidence in the standard drug discovery pipeline. The question is what must be done in order to demonstrate that this has real value or genuine potential value in modern drug discovery and development and for it to become more routinely used. It should not simply be a question of having confidence in modeling and simulation per se because they are already being used in pharmacokinetic/pharmacodynamic studies and also for compound and structure fitting in medicinal chemistry; the difference with systems biology is that modeling and simulation are being applied in a complex biological context in order to study and simulate the mechanisms at play.

\section{Q Why do you think that the pharmaceutical} industry, in general, is choosing not to embrace systems biology, despite its potential benefits?

I think that this is down to risk/benefit analysis - with the economics the way they are in industry right now, in addition to declining success rates and increased attrition failures, the focus from the point of view of the business needs to be on the bottom line. The question is, how do you mitigate the risk of investing in novel, potentially unproven technologies? I do not blame the pharmaceutical industry for being reserved about this and waiting to see whether this really is worthwhile. The evidence is mounting that these approaches should be applicable in the broadest sense, as I described earlier on, and I think that there needs to be adoption at least to some degree in industry. There are companies, such as AstraZeneca, Novartis and Roche, who have already adopted systems biology approaches to an extent, and may continue to do so, but systems biology is not yet broadly engaged in every single project at this stage. I think its potential value is recognized, but working out an economic model that would allow them to take the plunge is proving to be a challenge. In the future, I think we are increasingly going to see outsourcing of this kind of blue-skies, high-risk-highreward project to academic groups engaged in a range of collaborative ventures with industry, which may be on a one-to-one basis or, possibly, as projects under the umbrella of the European Innovative Medicines Initiative. 
Q You are currently leading a program known as the Virtual Liver Network; please could you briefly describe the project?

The Virtual Liver Network is a 5-year program funded by the German Federal Ministry of Education and Research. It is composed of over 200 contributing scientists in 36 independent institutions, running 44 projects involving 69 principle investigators across Germany, operating as a network, as opposed to a consortium. This network model operates as a large distributed project team in which individuals and groups in different institutions interact with one another on specific elements of a range of projects, rather than the consortium model, in which individual units contribute self-contained projects to a core theme. The model emulates the multidisciplinary project teams that are typical of drug discovery projects only in the sense that it is on a national scale as part of a $€ 50$ million program. The aim is to tackle an area of modeling in the biosciences that has not really been looked at, and that is to create a model, or series of models, that operate across scales of time and space in order to represent the function of an entire organ, in our case the 'multiscale' modeling of the liver. This involves the creation of models that come together in order to give a representation of liver function and dysfunction at the molecular level through all levels of organization in the organ and then through to function represented within the entire organism. Essentially, we have a direct line of sight from studies at the molecular and cellular level through to volunteer studies in clinical pharmacology units and surgical studies in patients. This helps us to acquire the quantitative data that are needed in order to inform the models that are being developed. We have succeeded in generating evidence of multiscale modeling at a limited level in tissues and cells, but there is a lot more to do. The focus of the program is broadly on three areas of clinical relevance: steatosis (fatty liver disease); the capacity of the liver to regenerate after injury; and inflammation in the liver. We also have a fourth area that looks at design principles in the liver and how liver structure and function has an impact on cellular function and the importance of this during the period of regeneration, so that when the liver regenerates, it does not regenerate in a disorganized fashion. The Virtual Liver Network is a flagship program of the German government and we have had a number of successes so far. We are beginning to show the power of systems biology approaches, but more importantly, we can show how modeling and simulation can be applied in order to address clinical needs. It is also a demonstration of how we can tackle 'big science' in biology in the way that engineers and physicists tend to, and that within biology, we can actually tackle big questions if we have the right approach, the right financial backing and the means for encouraging collaboration between industry and academia.

Q In what way do you think your work at the Virtual Liver Network will impact drug discovery \& development? Will it lead to more personalized medicine for patients?

If we think of systems biology as a new toolbox for applying mathematical and engineering principles in order to understand the dynamics of biological complexity, then it can be positioned in all sorts of areas to help us tackle challenges in the classical linear, reductionist approach that we have in drug discovery and development at the moment. At the Virtual Liver Network, we are increasingly working with the application of these approaches in the clinic and, if we are fortunate enough to secure further funding, the next phase will be to implement the approaches that we have developed, which have been driven by clinical imperatives. I think that this is a very exciting possibility, as it offers the opportunity to help clinicians improve the decisions that they make using information generated from dynamic models. I believe there is a very real possibility of achieving this to some degree in the next 5 years. Similar approaches are already operating at a physiological, physics-driven level in Auckland and in Sheffield, where models are being used to inform surgical planning. I think if we are less worried about labeling and pigeon-holing things into titles and disciplines, and instead simply think about the tools that we are producing and how they could be useful in tackling the problems that we face, then we are going to be successful.

\section{“...systems biology and systems medicine together provide a toolbox that I see as representing 21 st century physiology..."}

In terms of personalized medicine, my view is that it is very difficult to target therapies to an individual's needs if we cannot understand how a compound is going to operate in that individual as a whole - this requires a system-level understanding. We also need to understand that when we are giving a drug to an elderly patient, they may already be taking five or six compounds for other comorbidities, so a reductionist approach based on the current cell and animal model platforms is going to be a struggle. In order to improve our understanding, we need to employ a more holistic, integrated view of how systems operate dynamically. I think that systems biology and systems medicine together provide a toolbox that I see as representing 21st century physiology, rather than anything else. 
I do not see the ambitions of personalization and population segmentation as being competitors to the systems biology/systems medicine agenda, but rather they relate to all of these interests as being interdependent. I also believe that they are essential if we are going to succeed in understanding disease and how to treat complex diseases, especially in the aging population, which is currently what is crippling healthcare budgets.

\section{Q In what ways specifically is the virtual liver being used to influence early drug research?}

Currently, we are looking at the application of these approaches in a systems pharmacology setting, specifically at the role of variation in transporters in drug metabolism and in the cytochrome enzymes. We are also focusing on how we can use physiology-based pharmacokinetic models that are integrated into the overall functional view that we have from the other modeling experiments in the virtual liver, and using these to create predictions that can be used at a population level in order to inform a better positioning of drugs. This has already been done in prototyping experiments relating to OATP1B1 transporter and statins, the results of which have been published. In terms of other areas, we are also looking at integrating our models with the output in volunteers and patients who are exposed to a cocktail of well-known, wellmarketed drugs under ethically cleared studies. We have a very clear view of the importance of the liver in dealing with therapeutics and how interindividual variation can have an impact on how an individual responds to a particular therapeutic. So far, I would not say that we have had any major results, but we have certainly had some successes, demonstrating that these approaches have a role to play, and we have produced a number of strong publications in highranking journals. I am in no doubt that this is a very tractable area for future studies and that it warrants much greater attention.

\section{Q How do you anticipate that the field of systems biology will progress in the next \\ $5-10$ years?}

If the systems biology toolbox continues to be used, I believe that we will see great strides in improving decision-making by reducing the error bars in those decisions, as well as in terms of incremental benefits to the way things are done, if it is integrated into routine practice over time. I am sure that we will see some successes, as we have already seen, such as the one I mentioned earlier involving Merrimack Pharmaceuticals. I am sure that there will be more examples of successes in drug positioning in relation to development projects in the clinic. For me, systems biology is the logical step to follow on from the genome program, which generated, and continues to generate, a mass of important, detailed data and loads of information, all of which now needs to be integrated into an holistic view. The natural home for integration is, in my opinion, a systems-wide view built on the strengths of mathematical modeling and simulation strategies.

\section{Q Do you also hope to see systems biology} principles being further applied in the pharmaceutical industry in the future?

This is something that I have been advocating since 2003. Giulio Superti-Furga from the Centre for Molecular Medicine of the Austrian Academy of Sciences and I ran a workshop specifically focused on trying to come up with proposals for the application of systems biology in the drug discovery and development pipeline. We published a commentary article in Nature in October 2008 that detailed the broad outcomes of that workshop. This is something that I have been passionate about for a very long time. It has been the subject of many discussions and symposia, but it is still not being adopted routinely. The truth of the matter is that this really needs to be given enough time, investment and, most importantly, practical application in order to be tested and to prove itself within an industrial setting. Personally, I do not think that we will fail as long as we avoid treating systems biology as a separate discipline and a new area of research. This is about the application of tools that are well-established in engineering, but not routinely used in biology - mainly those of mathematics and engineering - bringing these into the fold and helping us to understand the dynamics of the complex biological systems we are studying.

Q What words of advice do you have for a young scientist envisioning a career in systems biology with a view to advancing pharmaceutical R\&D?

Some 7 years ago, I was asked a similar question by a man who was in a permanent position working within safety assessment. He was aware of the work we were doing in systems biology in our department and he came and asked whether he should apply for a $\mathrm{PhD}$ in systems biology. At that stage, AstraZeneca had a very close relationship with the new multidisciplinary institute in Manchester and we had just funded the first AstraZeneca Chair in Systems Biology in the University of Manchester. I told him that I could not see how the pharmaceutical industry, or indeed biology, could progress post-genome without this systems biology toolbox being adopted. It might take a long time, but I could not see how we 
could achieve what is necessary without being able to understand systems dynamics, and that means using mathematics, physics and engineering principles in a biological context. I did not advise this man one way or the other, but told him that he needed to consider whether his belief in the potential of systems biology was strong enough to leave a permanent job in order to go for a PhD studentship, and then to move around on various research grants until finding another established position. In the end, he went and did his $\mathrm{PhD}$ and he is now working at a company that specializes in systems pharmacology. His was a very real question that put me on the spot. Given a similar question again now, I would respond with the same answer.

\section{Disclaimer}

The opinions expressed in this interview are those of the interviewee and do not necessarily reflect the views of Future Medicine Ltd.

\section{Financial \& competing interests disclosure}

A Henney gratefully acknowledges the support of the German Federal Ministry of Research (Bundesministerium für Bildung und Forschung) for the Flagship Program 'The Virtual Liver Network'. A Henney has no other relevant affiliations or financial involvement with any organization or entity with a financial interest in or financial conflict with the subject matter or materials discussed in the manuscript apart from those disclosed.

No writing assistance was utilized in the production of this manuscript. 